\title{
THE PRESSURE OF RADIATION ON A CYLINDRICAL OBSTACLE
}

\author{
By J. W. Nicholson, M.A., D.Sc. \\ [Received November 1st, 1911.-Read December 14th, 1911.-Revised version Received \\ January 10th, 1912.]
}

\section{Part I.}

IN an earlier paper * the pressure experienced by a reflecting spherule on which plane waves impinge was examined numerically, with special reference to the maximum value of this pressure corresponding to an obstacle whose radius is of the same order of magnitude as the wave length of the incident radiation. The determination of a corresponding maximum, when it exists, for obstacles which are not spherical is a matter of importance; but the mathematical difficulties are so formidable that no solution has yet been given. As a preliminary to a more complete knowledge of the pressure which can be experienced by non-spherical bodies, the present paper takes up the only other case in which an analysis on similar lines to that used for a spbere can be employed profitably. This is the two-dimensional problem of the circular cylinder whose length is sufficiently great in comparison with a radius of the cross-section and with the wave length of light.

The general basis of the investigation is the electromagnetic theory. It appears that all possible cases can be built up from two when the incident waves are travelling perpendicularly to the axis of the cylinder. The two cases are those in which (1) the electric force and (2) the magnetic force are respectively parallel to that axis. In Part I of the paper we propose to consider the first case in detail. The second case, which is somewhat more complicated, is investigated in Part II.

Let a set of cylindrical coordinates $(r, \theta, z)$ be chosen, where $z$ is parallel to the axis, so that $r$ and $\theta$ are polar coordinates in the plane of a normal section of the cylinder. The problem being one of symmetry, none of the quantities concerned will be functions of $z$. If the wave length be $2 \pi / k$, and if the incident waves are travelling along a direction $x$, the initial direction from which $\theta$ is measured, the magnetic 
force in the incident waves may be taken as $e^{i k x}$, ignoring a time factor, acting along the direction $y$ perpendicular to $x$ and $z$. Moreover,

$$
x=r \cos \theta, \quad y=r \sin \theta .
$$

The waves are incident on the cylinder on the side for which $x$ is positive.

If the cylinder is a perfect reflector, the magnetic force at its surface must be tangential and the electric force normal. A disturbance will therefore be thrown off, in which the radial magnetic force at the surface $r=a$ ( $a$ being the radius of the cylinder) will be equal and opposite to that of the incident waves. The latter is, of course,

$$
\frac{y}{r} e^{i k \cdot c} \text { or } \sin \theta . e^{i l \cdot r \cos \theta},
$$

which may be expressed by a well known theorem ascribed by Nielsen* to Hansen, but probably used earlier by Jacobi, in the form

$$
\sin \theta \cdot J_{0}(k r)+2 \sin \theta \Sigma_{1}^{\infty} i^{n} J_{n}(k r) \cos n \theta,
$$

where $J_{n}(k r)$ is the usual Bessel function of argument $k r$ and order $n$, finite at the origin, or on the axis of the cylinder. This series may he written in the form

$$
\sin \theta\left(J_{0}+J_{2}\right)+i \sin 2 \theta\left(J_{1}+J_{9}\right)+i^{2} \sin 3 \theta\left(J_{2}+J_{4}\right)+\ldots,
$$

and, by the use of a recurrence formuli for the Bessel functions, this becomes

$$
a_{1}=\frac{2}{k r} \Sigma_{1}^{\infty} i^{n-1} n \sin n \theta . J_{n}(k \cdot r) .
$$

This might also have been obtained by differentiating the original series with respect to $\theta$. The electric force along the axis is

$$
R=V e^{i k x}=V J_{0}(k r)+2 V \Sigma_{1}^{\infty} i^{n} J_{n}(k r) \cos n \theta .
$$

Let us now consider the general equations of the external electromagnetic field. If $(P, Q, R)$ and $(a, b, c)$ be the components of the electric and magnetic vectors along the directions at any point in which $r, \theta$, and $z$ respectively increase, then, by the cylindrical form of the circuital electromagnetic relations,

$$
\left.\begin{array}{ll}
-r \dot{a}=\frac{\partial R}{\partial \theta}-r \frac{\partial Q}{\partial z}, & \frac{r \dot{P}}{V^{2}}=\frac{\partial c}{\partial \theta}-r \frac{\partial \partial}{\partial z} \\
-\dot{b}=\frac{\partial P}{\partial z}-\frac{\partial R}{\partial r}, & \frac{r \dot{Q}}{V^{2}}=\frac{\partial a}{\partial z}-\frac{\partial c}{\partial r} \\
-r \dot{c}=\frac{\partial}{\partial r}(Q r)-\frac{\partial P}{\partial \theta}, & \frac{r \dot{R}}{V^{2}}=\frac{\partial}{\partial r}(b r)-\frac{\partial a}{\partial \theta}
\end{array}\right\},
$$


with the solenoidal conditions

$$
\frac{\partial}{\partial r}(a r)+\frac{\partial b}{\partial \theta}+r \frac{\partial c}{\partial z}=\frac{\partial}{\partial r}(P r)+\frac{\partial Q}{\partial \theta}+r \frac{\partial R}{\partial z}=0,
$$

where $V$ is the velocity of disturbances in the outer medium. For the present problem, $\partial / \partial z \equiv 0$, and therefore, by the solenoidal conditions, functions $\phi$ and $\psi$ exist such that

$$
\left.\begin{array}{ll}
P=-\frac{\partial \phi}{r \partial \theta}, & Q=\frac{\partial \phi}{\partial r} \\
a=-\frac{\partial \psi}{r \partial \theta}, & b=\frac{\partial \psi}{\partial r}
\end{array}\right\} .
$$

If the disturbance has a wave-length $2 \pi / k$, it will involve a time factor $e^{i k v t}$, and we accordingly write $\partial / \partial t=i k V$, and derive, on reduction,

$$
R=i k V \psi, \quad c=i k \phi / V,
$$

where $\phi$ and $\psi$ appear as solutions of the equation

$$
\frac{\partial^{2} \psi}{\partial r^{2}}+\frac{1}{r} \frac{\partial \psi}{\partial r}+\frac{1}{r^{2}} \frac{\partial^{2} \psi}{\partial \theta^{2}}+k^{2} \psi=0 .
$$

For the most general vibration of a given period there is no essential relation between $\phi$ and $\psi$. Thus, the most general vibration of given period in or about a system of coaxial cylinders of circular section, independent of $z$, consists of a superposition of two others, in one of which the electric force, and in the other the magnetic force, is parallel to the axis at all points. This is a special case of a more general result true for orthogonal sets of surfaces of revolution.

The general solution of (7) finite at the origin is

$$
\psi=\Sigma_{0}^{\infty} A_{n} J_{n}(k r) \cos n \theta .
$$

Let us consider the case in which the incident waves have no magnetic component parallel to the axis. Then a complete solution may be obtained in terms of functions of type $\psi$ only, the electric force in both incident and scattered waves having a component $R$ only parallel to the axis, and the magnetic force having components $(a, b)$ in the plane of the circular section at any point.

The function $\psi$ for the incident waves is, by (2) and (6), of the form

$$
\psi_{1}=-\frac{i}{k} \Phi_{0}(k r)+\frac{2}{k} \Sigma_{1}^{\infty} i^{n-1} J_{n}(k r) \cos n \theta,
$$

leading by (4) at once to the proper formula (1). 
If $K_{n}(k r)$ be the corresponding function of distance for the scattered waves, we may write, for these waves,

$$
\psi_{2}=-\frac{i a_{0}}{k} K_{0}(k r)+\frac{2}{k} \Sigma_{1}^{\infty} i^{n-1} a_{n} K_{n}(k r) \cos n \theta,
$$

and $a_{n}$ may be determined from the general surface condition of the continuity of tangential components of electric and magnetic force. For a perfect reflector it is sufficient to make $a$ or $-\partial \psi / r \partial \theta$ and $R$ or $i k V \psi$ vanish at the surface. Both conditions are secured if $\psi$ itself vanishes, and therefore if

$$
\left.\begin{array}{l}
a_{0}=-J_{0}(k a) / K_{0}(k a) \\
a_{n}=-J_{n}(k a) / K_{n}(k \cdot a)
\end{array}\right\}
$$

$a$ now denoting the radius, and the form of $\psi=\psi_{1}+\psi_{2}$ beconres

$$
\begin{aligned}
\psi=-\frac{i}{k}\left\{J_{0}(k r)-J_{0}(k a) K_{0}(k r) / K_{0}(k a)\right\} \\
+\frac{2}{k} \Sigma_{1}^{\infty} i^{n-1}\left\{J_{n}(k v)-J_{n}(k a) K_{n}(k r) / K_{n}(k a)\right\} \cos n \theta .
\end{aligned}
$$

Let $Y_{n}(x)$ be the second solution of Bessel's equation of order $n$ defined by *

$$
Y_{n}(x)=\left\{\frac{\partial J_{n}}{\partial n}-(-)^{n} \frac{\partial J_{-i n}}{\partial n}\right\}_{n=\text { integer }} .
$$

Then

$$
\frac{K_{n}(k r)}{K_{n}(k a)}=\frac{Y_{n}(k r)+i \pi J_{n}(k r)}{Y_{n}(k a)+i \pi J_{n}(k a)} .
$$

The electric force vanishes at the surface. The magnetic component is only $b$ or $\partial \psi / \partial r$, and becomes on the surface

$$
\begin{aligned}
b=-i\left\{\frac{J_{0}^{\prime}(k a) Y_{0}(k a)-Y_{0}^{\prime}(k a) J_{0}(k a)}{Y_{0}(k a)+i \pi J_{0}(k a)} ;\right. \\
\left.\quad+2 \Sigma_{1}^{\infty} i^{n-1} ! \frac{J_{n}^{\prime}(k a) Y_{n}(k a)-Y_{n}^{\prime}(k a) J_{n}(k a)}{Y_{n}(k a)+i \pi J_{n}(k a)}\right\} \cos n \theta .
\end{aligned}
$$

The two kinds of Bessel function are related by the identity

$$
Y_{n}(x) J_{n}^{\prime}(x)-J_{n}(x) Y_{n}^{\prime}(x)=-2 / x,
$$

and the surface value of $b$ becomes, inserting the time factor,

$$
b=\frac{2 i}{M_{0} k a} e^{i k V t-i \eta_{0}}-\frac{4}{k a} \sum_{1}^{\infty} i^{n-1} \frac{\cos n \theta}{M_{n}} e^{i k v t-i \eta_{n},}
$$

* The referee points out that this definition may cause confusion. It was adopted mainly to keep the notation in line with that of earlier papers. The present function is more usually given a factor in English work, and is identical with $-2 K_{n}$, where the latter symbol is used in the sense of Heine. 
where

$$
\left.\begin{array}{rl}
M_{n}^{2} & =Y_{n}^{2}(k a)+\pi^{2} J_{n}^{2}(k a) \\
\tan \eta_{n} & =\pi J_{n}(k a) / Y_{n}(k a)
\end{array}\right\} .
$$

\section{Determination of the Pressure.}

Let $E$ be the energy per unit volume of the incident radiation, half electric and half magnetic. Then the pressure on a unit length of the cylinder parallel to its axis has a resultant action along the direction of propagation of the incident waves, given by

$$
p=E \iint b^{2} \cos \theta d S
$$

the surface integral being taken over the unit length. This follows at once from the Maxwellian stress system, the electric force being zero. Expressed as a line integral round the circular section, the formula becomes

$$
p=2 a E \int_{c}^{\pi} b^{2} \cos \theta d \theta .
$$

In this calculation the average value of $b^{2}$ is needed for the determination of the motive pressure; that is, the average value over a sufficiently large time, which neglects the periodic terms. It is a familiar relation that the average value of the square of a complex $b$ is given by $\frac{1}{2}|b|^{2}$ or $\frac{1}{2} b \vec{b}$, where $\vec{b}$ is the conjugate complex. In the present case this average becomes $B$, where

$$
\begin{aligned}
2 B=\frac{4}{k^{2} a^{2}} \frac{1}{M_{0}^{2}} & +\frac{16}{k^{2} a^{2}} \Sigma_{1}^{\infty} \frac{\cos ^{2} n \theta}{M_{n}^{2}}+\frac{16}{k^{2} a^{2}} \frac{1}{M_{0}} \Sigma_{1}^{\infty} \frac{\cos n \theta}{M_{n}} \cos \left(\eta_{0}-\eta_{n}+\frac{n \pi}{2}\right) \\
& +\frac{32}{k^{2} a^{2}} \Sigma_{1}^{\infty} \Sigma_{1}^{\infty} \frac{\cos r \theta \cos s \theta}{M_{r} M_{s}} \cos \left\{\left(\eta_{r}-\eta_{s}+(s-r) \frac{\pi}{2}\right\},\right.
\end{aligned}
$$

where the double summation excludes equal values of $r$ and $s$.

Now for all integral values of $n, r, s$,

$$
\begin{gathered}
\int_{0}^{\pi} \cos ^{2} n \theta \cos \theta d \theta=0, \\
\int_{0}^{\pi} \cos r \theta \cos s \theta \cos \theta d \theta=\frac{1}{2} \int_{0}^{\pi}\{\cos (r+s) \theta+\cos (r-s) \theta\} \cos \theta d \theta .
\end{gathered}
$$

The first integral vanishes because $r+s$ is necessarily greater than unity. The second also vanishes unless $r-s=1$, in which case

$$
\int_{0}^{\pi} \cos r \theta \cos s \theta \cos \theta d \theta=\frac{\pi}{4} \text {. }
$$


Moreover, when $n=1$,

$$
\int_{0}^{\pi} \cos n \theta \cos \theta d \theta=\frac{\pi}{2}
$$

being zero for other integral values of $n$.

$$
\text { Thus } \quad \begin{aligned}
p & =2 a E \int_{0}^{\pi} B \cos \theta d \theta \\
& =-\frac{8 \pi a E}{k^{2} a^{2}} \Sigma_{0}^{\infty}\left(M_{n} M_{n+1}\right)^{-1} \sin \left(\eta_{n}-\eta_{n+1}\right),
\end{aligned}
$$

all the terms falling into line with one another.

But $\quad-\sin \left(\eta_{n}-\eta_{n+1}\right)=\pi\left(Y_{n} J_{n+1}-J_{n} Y_{n+1}\right) / M_{n} M_{n+1}$, by (18), and by an identical relation

$$
Y_{n} J_{n+1}-J_{n} Y_{n+1}=2 / k a,
$$

we deduce

$$
\frac{p}{2 a E}=\frac{8 \pi^{2}}{k^{3} a^{8}} \Sigma_{0}^{\infty} M_{n}^{-2} M_{n+1}^{-2} ;
$$

or if $z$ denotes $k u$, the argument of the Bessel functions,

$$
\frac{p}{2 a E}=\frac{8 \pi^{2}}{z^{3}} \Sigma_{0}^{\infty}\left(Y_{n}^{2}+\pi^{2} J_{n}^{2}\right)^{-1}\left(Y_{n+1}^{2}+\pi^{2} J_{n+1}^{2}\right)^{-1},
$$

gives the pressure per unit length of the cylinder. This result is of considerable elegance.

\section{General Remarks.}

In the first place, before making a detailed investigation of the formula just obtained, we may shew that it approaches the correct limiting value when the diameter of the obstacle increases indefinitely.

On the usual theory of pressure, a plate of a reflecting body, on which radiation falls at an angle $\theta$ to the normal, experiences a normal pressure $2 E \cos ^{2} \theta$ per unit area, where $E$ is energy in a unit volume of the

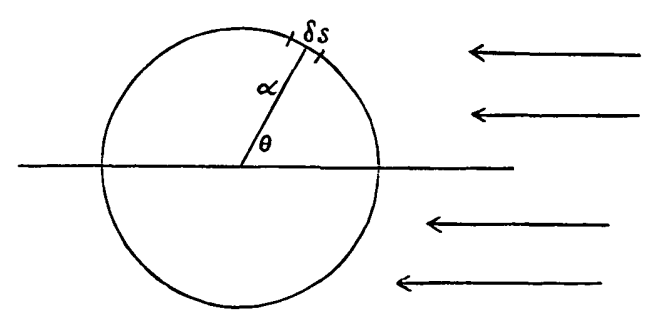

incident beam. If the cylinder has a large diameter, and attention is 
confined to a unit length along the axis, the pressure on the plate of unit length and breadth $\delta s$, an element of arc of the circular section, becomes $2 E \delta s \cos ^{2} \theta$. This acts towards the axis of the cylinder and has a component $2 E \delta s \cos ^{3} \theta$ parallel to the incident rays. The resultant pressure in this direction is obtained by integration over the unshadowed part, or just one half, of the surface and becomes

$$
p=\int_{-3 \pi}^{3 \pi} 2 E \cos ^{3} \theta \cdot a d \theta=\frac{8}{3} a E
$$

per unit length of cylinder.

Consider now the accurate formula

$$
\frac{p}{2 a E}=\frac{8 \pi^{2}}{z^{3}} \Sigma_{0}^{\infty} \frac{1}{\left(Y_{n}^{2}+\pi^{2} J_{n}^{2}\right)\left(Y_{n+1}^{2}+\pi^{2} J_{n+1}^{2}\right)} .
$$

Writing

$$
\left.\begin{array}{l}
J_{n}(z)=\left(\frac{2 R_{n}}{\pi z}\right)^{\frac{1}{2}} \sin \phi_{n} \\
Y_{n}(z)=-\left(\frac{2 \pi R_{n}}{z}\right)^{\frac{1}{2}} \cos \phi_{n}
\end{array}\right\},
$$

where the functions $R_{n}$ and $\phi_{n}$ have been examined in detail in earlier papers, ${ }^{*}$ then

$$
\frac{p}{2 a E}=\frac{2}{z} \Sigma_{0}^{\infty} \frac{1}{R_{n} R_{n+1}} \text {. }
$$

Now, when $z$ is large, and $n$ is not so large that $z-n$ is of so low an order as $z^{\frac{3}{3}}$,

$$
R_{n}=z /\left(z^{2}-n^{2}\right)^{\frac{1}{2}}
$$

approximately, and the number of terms of the series for which this occurs is effectively $z$.

When $n-z$ or $z-n$ is of order $z^{\frac{1}{3}}$ or less, the number of terms thus concerned being also of this order, $R_{n}$ and $R_{n+1}$ are of order $z^{k}$. The sum of such terms is therefore of order $z^{-\frac{1}{3}}$ at most in the series $\Sigma\left(R_{n} R_{n+1}\right)^{-1}$ and is negligible in comparison with the earlier terms, which have a sum of order $z$.

When $n$ is greater than $z$, and $n-z$ is of higher order than $z^{\ddagger}$, the functions $R_{n} R_{n+1}$ become large in comparison with any power of $z$, and the sum of the terms involving them is not significant + .

- Vide Phil. Mag., February, 1910.

† A similar problem is discussed in Phil. Mag., April, 1910. 
These points are all illustrated very clearly in the numerical tables which follow. Finally, then, when $z$ is large, we may .write

$$
\frac{p}{2 a E}=\frac{2}{z^{3}} \sum_{n=0}^{z}\left(z^{2}-n^{2}\right)^{\frac{1}{2}}\left[z^{2}-(n+1)^{2}\right]^{\frac{3}{2}} .
$$

In determining the leading term in the sum of this series, expressed in inverse powers of $z$, we may replace it by an integral in the usual way, writing $n / z=x$ and $z^{-1}=d x$, so that

$$
\begin{gathered}
\overline{2} \frac{p}{a E}=2 \int_{1}^{1}\left(1-x^{2}\right) d x=\frac{1}{3}, \\
p=\frac{\kappa}{3} a E .
\end{gathered}
$$

or

This is in accordance with (28), and the formula (27) therefore tends to the proper limiting value for a large obstacle.

The function $R_{n}$ used above is not identical with the $R_{n}$ of the earlier paper on radiation pressure. The latter, in the present notation, would be $R_{n+\frac{1}{1}}$ or $R_{m}, m$ denoting $n+\frac{1}{2}$, as throughout that paper. The functions $R_{n}$ and $\phi_{n}$ possess the properties

$$
\left.\begin{array}{c}
R_{n+1}=R_{n-1}+\frac{4 n^{2}}{z^{2}} R_{n}-\frac{2 n}{z} \sqrt{ }\left(R_{n} R_{n-1}-1\right) \\
\sin \left(\phi_{n}-\phi_{n+1}\right)=\left(R_{n} R_{n+1}\right)^{-1}
\end{array}\right\},
$$

the proofs of these relations in the earlier paper not being confined to the case in which $n$ is half an odd integer.

\section{The Small Obstacle.}

It is also convenient, before proceeding to tabulation, to obtain a formula for the pressure on a very thin obstacle of cylindrical shape, due to its scattering action on the incident radiation. For this purpose attention may be confined to the first term of (27), and we may also write

$$
\left.\begin{array}{l}
Y_{0}(z)=2\left\{\log \frac{z}{2}+\gamma\right\} \\
J_{0}(z)=1, \quad Y_{1}(z)=-2 / z, \quad J_{1}(z)=0
\end{array}\right\},
$$

where $\gamma$ is Euler's constant. Thus

$$
\frac{p}{2 a E}=\frac{8 \pi^{2}}{z^{3}} \frac{z^{2}}{4} \frac{1}{\pi^{2}+4\left(\gamma+\log \frac{1}{2} z\right)^{2}} ;
$$


or, finally, since $z=2 \pi a / \lambda$,

$$
p=2 \lambda \pi E /\left\{\pi^{2}+4(\gamma+\log \pi a / \lambda)^{2}\right\},
$$

and we note that the pressure tends to zero with the radius of the obstacle. The ratio $p / \frac{8}{3} a E$, on the contrary, tends to infinity, exhibiting a marked difference from the behaviour of the corresponding ratio in the case of the sphere.

\section{Numerical Results.}

A large amount of numerical work is necessary in the tabulation of the formula (27), and a fairly complete account of it is given, as many of the intermediate figures are useful for other purposes.

In the first two tables the values of the Bessel functions are given. The functions of the logarithmic type $Y_{n}(z)$ have only been tabulated hitherto for the cases $n=0$ and $n=1$. The values for these cases have been taken from B. A. Smith's tables, ${ }^{*}$ and those for higher values of $n$ have been deduced by the recurrence formula

$$
Y_{n+1}(z)=\frac{2 n}{z} Y_{n}(z)-Y_{n-1}(z) .
$$

The notation is different from that of Smith's tables. His function $(\log 2-\gamma) J_{n}(z)-Y_{n}(z)$ is identical with the function $-\frac{1}{2} Y_{n}(z)$ of this paper. As Smith's tables are restricted to a few significant figures, the last figure in the present table for $Y_{n}(z)$ will sometimes be incorrect to the extent of a unit.

The functions of type $J_{n}(z)$ involved less calculation. $J_{0}(z)$ and $J_{1}(z)$ have been taken from Meissel's tables. $t$ For integer values of $z$ the functions of higher orders are also taken from these tables. But, for non-integral values such as $z=1 \cdot 5$, it was necessary to calculate them from $J_{0}$ and $J_{1}$ by the recurrence formula

$$
J_{n+1}(z)=\frac{2 n}{z} J_{n}(z)-J_{n-1}(z) .
$$

* Phil. Mag., 1898, Vol. 1, p. 122. They are reprinted by Jahnke and Emde, Functionentafeln, Leipzig (B. G. Teubner).

$\dagger$ Gray and Matherws's Treatise on Bessel Functions. 
TABLE 1.

\begin{tabular}{|c|c|c|c|c|c|c|c|}
\hline$z$ & $\cdot 1$ & $\cdot 4$ & $\cdot 5$ & $\cdot 6$ & .8 & $\cdot 9$ & 1.0 \\
\hline$J_{11}$ & .9975 & $\cdot 9604$ & $\cdot 9385$ & $\cdot 9120$ & $\cdot 8463$ & $\cdot 8075$ & .7652 \\
\hline$J_{1}$ & .0499 & $\cdot 1960$ & $\cdot 2423$ & .2867 & $\cdot 3688$ & $\cdot 4059$ & $\cdot 4401$ \\
\hline$J_{2}$ & .0012 & 0197 & .0306 & .0437 & .0758 & .0946 & $\cdot 1149$ \\
\hline$J_{3}$ & $<10^{-3}$ & $\cdot 0013$ & .0026 & .0044 & .0102 & .0144 & 0196 \\
\hline$-Y_{0}$ & $4 \cdot 820$ & $1 \cdot 904$ & $1 \cdot 397$ & $\cdot 9692$ & .2726 & -.0176 & -.2772 \\
\hline$-Y_{1}$ & $20 \cdot 29$ & $5 \cdot 595$ & $4 \cdot 623$ & 3.960 & 3.073 & $2 \cdot 743$ & $2 \cdot 454$ \\
\hline$-Y_{2}$ & $401 \cdot 0$ & 26.07 & $17 \cdot 095$ & $12 \cdot 23$ & $7 \cdot 410$ & $6 \cdot 113$ & $5 \cdot 186$ \\
\hline$-Y_{: 3}$ & $>10^{3}$ & $255 \cdot 1$ & $132 \cdot 1$ & $77 \cdot 57$ & 33.98 & $24 \cdot 43$ & 18.29 \\
\hline$-Y_{4}$ & $>10^{4}$ & $>10^{3}$ & $>10^{3}$ & 763.5 & $247 \cdot 4$ & $156 \cdot 7$ & $104 \cdot 5$ \\
\hline
\end{tabular}

In this and the following table, the values of the $Y_{n}$ functions have been checked as far as three figures in the last function of any series by means of the relation

$Y_{n} J_{n+1}-J_{n} Y_{n+1}=2 / z$.

TABLE 2.

\begin{tabular}{|c|c|c|c|c|c|c|c|c|c|}
\hline$z$ & $1 \cdot 1$ & $1 \cdot 2$ & $1 \cdot 5$ & 2 & $2 \cdot 5$ & 3 & 4 & 5 & 6 \\
\hline$J_{1}$ & $\cdot 7196$ & $\cdot 6711$ & $\cdot 5118$ & $\cdot 2239$ & $-\cdot 0484$ & $-\cdot 26005$ & $-\cdot 3971$ & $-\cdot 1776$ & $\cdot 1506$ \\
\hline$J_{1}$ & $\cdot 4709$ & $\cdot 4983$ & $\cdot 5579$ & $\cdot 5767$ & $\cdot 4971$ & $\cdot 3391$ & $-\cdot 0660$ & $-\cdot 3276$ & $-\cdot 2767$ \\
\hline$J_{2}$ & $\cdot 1366$ & $\cdot 15935$ & $\cdot 2321$ & $\cdot 3528$ & $\cdot 4461$ & $\cdot 4861$ & $\cdot 3641$ & $\cdot 0466$ & $-\cdot 2429$ \\
\hline$J_{3}$ & $\cdot 0257$ & $\cdot 0329$ & $\cdot 06095$ & $\cdot 1289$ & $\cdot 2166$ & $\cdot 3091$ & $\cdot 4302$ & $\cdot 3648$ & $\cdot 1148$ \\
\hline$J_{4}$ & $\cdot 0036$ & $\cdot 005$ & $\cdot 0117$ & $\cdot 0340$ & $\cdot 0738$ & $\cdot 1320$ & $\cdot 2811$ & $\cdot 3912$ & $\cdot 3576$ \\
\hline$J_{i}$ & $<10^{-3}$ & $\cdot 0006$ & $\cdot 0016$ & $\cdot 0070$ & $\cdot 0195$ & $\cdot 0430$ & $\cdot 1321$ & $\cdot 2611$ & $\cdot 3621$ \\
\hline$J_{1 i}$ & $<10^{-4}$ & $<10^{-1}$ & $\cdot 0004$ & $\cdot 0012$ & $\cdot 0042$ & $\cdot 0114$ & $\cdot 0491$ & $\cdot 13105$ & $\cdot 2458$ \\
\hline$J_{7}$ & $<10^{-5}$ & $<10^{-5}$ & $<10^{-3}$ & $<10^{-3}$ & $10^{-3}$ & $\cdot 0025$ & $\cdot 0152$ & $\cdot 0534$ & $\cdot 1296$ \\
\hline$J_{3}$ & $<10^{-6}$ & $<10^{-6}$ & $<10^{-4}$ & $<10^{-4}$ & $<10^{-3}$ & $<10^{-3}$ & $\cdot 0040$ & $\cdot 0184$ & $\cdot 0565$ \\
\hline$-I_{11}$ & $-\cdot 5096$ & $-\cdot 7166$ & $-1 \cdot 2016$ & $-1 \cdot 6034$ & $-1 \cdot 5648$ & $-1 \cdot 1840$ & $+\cdot 0532$ & $+\cdot 9692$ & $+\cdot 9054$ \\
\hline$-I_{1}$ & $2 \cdot 193$ & $1 \cdot 951$ & $1 \cdot 295$ & $\cdot 3364$ & $-\cdot 4586$ & $-1 \cdot 020$ & $-1 \cdot 2502$ & $-\cdot 4646$ & $\cdot 5498$ \\
\hline$-Y_{2}$ & $4 \cdot 497$ & $3 \cdot 969$ & $2 \cdot 929$ & $1 \cdot 940$ & $1 \cdot 198$ & $\cdot 5040$ & $-\cdot 6783$ & $-1 \cdot 155$ & $-\cdot 7221$ \\
\hline$-Y_{3}$ & $14 \cdot 16$ & $11 \cdot 28$ & $6 \cdot 515$ & $3 \cdot 541$ & $2 \cdot 375$ & $1 \cdot 692$ & $\cdot 572$ & $-\cdot 460$ & $-1 \cdot 031$ \\
\hline$-Y_{4}$ & $72 \cdot 74$ & $52 \cdot 42$ & $23 \cdot 13$ & $8 \cdot 69$ & $4 \cdot 50$ & $2 \cdot 88$ & $1 \cdot 536$ & $\cdot 604$ & $-\cdot 3091$ \\
\hline$-Y_{5}$ & 515 & 338 & $116 \cdot 8$ & $31 \cdot 22$ & $12 \cdot 03$ & $5 \cdot 99$ & $2 \cdot 50$ & $1 \cdot 426$ & $\cdot 619$ \\
\hline$-Y_{6}$ & $>10^{3}$ & $>10^{3}$ & 755 & $147 \cdot 4$ & $43 \cdot 62$ & $17 \cdot 08$ & $4 \cdot 715$ & $2 \cdot 247$ & $1 \cdot 341$ \\
\hline
\end{tabular}

sE1. 2. VOL. 11 . No. 1136 . 
The following additional values have also been employed :-

$$
\begin{array}{lll}
Y_{7}(3)=-62 \cdot 3, & Y_{7}(4)=-11 \cdot 645, & Y_{7}(5)=-3 \cdot 968, \\
Y_{7}(6)=-2 \cdot 063, & \\
Y_{8}(4)=-36 \cdot 04, & Y_{8}(5)=-8 \cdot 863, & Y_{8}(6)=-3 \cdot 473, \\
Y_{9}(\tilde{0})=-24 \cdot 395, & Y_{9}(6)=-7 \cdot 198, \\
Y_{10}(6)=-18 \cdot 12 . & \\
\text { Let } & \rho_{n}=Y_{n}^{2}(z)+\pi^{2} J_{n}^{2}(z), \\
& \rho_{n}=\frac{2 \pi}{z} R_{n} .
\end{array}
$$

Let

\begin{tabular}{|c|c|c|c|c|c|c|c|}
\hline$z$ & $P_{11}$ & $\rho_{1}$ & $\rho_{2}$ & $\rho_{3}$ & $\rho_{4}$ & $\rho_{i}$ & $\rho_{i ;}$ \\
\hline $1 \cdot 1$ & $5 \cdot 3707$ & 6.999 & $20 \cdot 409$ & $200 \cdot 5$ & 5291 & $26 \cdot 10^{4}$ & $>10^{i}$ \\
\hline $1 \cdot 2$ & 4.9591 & $6 \cdot 257$ & 16.003 & $127 \cdot 25$ & 2746 & $13 \cdot 10^{4}$ & $>10^{i j}$ \\
\hline 1.5 & 4.0304 & $4 \cdot 750$ & $9 \cdot 108$ & $42 \cdot 50$ & $535 \cdot 0$ & $14 \cdot 10^{x}$ & $>10^{\circ}$ \\
\hline 2 & 3.0660 & $3 \cdot 396$ & 4.991 & $12 \cdot 72$ & $75 \cdot 52$ & $974 \cdot 4$ & $16 \cdot 10^{3}$ \\
\hline 2.5 & $2 \cdot 4715 \mid$ & $2 \cdot 649$ & $3 \cdot 399$ & $6 \cdot 103$ & $20 \cdot 32$ & $144 \cdot 8$ & $190: 3$ \\
\hline 3 & 2.0693 & $2 \cdot 175$ & $2 \cdot 570$ & $3 \cdot 806$ & $8 \cdot 466$ & 35.87 & $291 \cdot 7$ \\
\hline 4 & 1.5590 & $1 \cdot 606$ & 1.7685 & $2 \cdot 154$ & $3 \cdot 140$ & 6.425 & $22 \cdot 255$ \\
\hline 5 & $1 \cdot 2505$ & $1 \cdot 275$ & $1 \cdot 355$ & 1.525 & 1.875 & $2 \cdot 706$ & $5 \cdot 220$ \\
\hline 6 & 1.0436 & 1.058 & $1 \cdot 104$ & $\begin{array}{ll}1 & 1935\end{array}$ & $1 \cdot 358$ & $1 \cdot 677$ & $2 \cdot 394$ \\
\hline
\end{tabular}

so that

Then the values of $\rho_{\imath}$ are exbibited in the next two talles:-

TABLE 3.

\begin{tabular}{|c|c|c|c|c|c|c|c|}
\hline$z$ & $\cdot 1$ & $\cdot 4$ & $\cdot 5$ & $\cdot 6$ & $\cdot 8$ & $\cdot 9$ & $1 \cdot 0$ \\
\hline$\rho_{0}$ & $33 \cdot 050$ & $12 \cdot 728$ & $10 \cdot 643$ & $9 \cdot 148$ & $7 \cdot 143$ & $6 \cdot 436$ & $5 \cdot 856$ \\
\hline$\rho_{1}$ & $411 \cdot 75$ & $31 \cdot 679$ & $21 \cdot 950$ & $16 \cdot 490$ & $10 \cdot 786$ & $9 \cdot 151$ & $7 \cdot 933$ \\
\hline$\rho_{z}$ & $16 \cdot 10^{4}$ & $679 \cdot 6$ & $292 \cdot 2$ & $149 \cdot 6$ & $54 \cdot 96$ & $37 \cdot 46$ & $28 \cdot 20$ \\
\hline$\rho_{3}$ & $>10^{4}$ & $65 \cdot 10^{3}$ & $17 \cdot 10^{3}$ & 6017 & 1154 & $596 \cdot 6$ & $334 \cdot 5$ \\
\hline$\rho_{4}$ & $>10^{i}$ & $>10^{6}$ & $>10^{6}$ & $>10^{5}$ & $>5 \cdot 10^{4}$ & $25 \cdot 10^{3}$ & $11 \cdot 10^{3}$ \\
\hline
\end{tabular}

TABLE 4. 
1911.] The pressure of Radiation on a cylindrical obstacle.

The following additional values were used:-

$$
\begin{array}{ll}
\rho_{7}(4)=135 \cdot 6, & \rho_{7}(5)=15 \cdot 77, \quad \rho_{7}(6)=4 \cdot 121, \\
\rho_{8}(5)=78 \cdot 56, & \rho_{8}(6)=12 \cdot 09, \\
\rho_{9}(6)=51 \cdot 82, & \rho_{10}(6)=328 .
\end{array}
$$

The fifth and sixth tables exhibit the corresponding values of $1 / \rho_{n} \rho_{n+1}$

\begin{tabular}{|c|c|c|c|c|c|c|c|}
\hline$z$ & $\left(\rho_{0} \rho_{1}\right)^{-1}$ & $\left(\rho_{1} \mu_{2}\right)^{-1}$ & $\left(\rho_{2} \rho_{3}\right)^{-1}$ & $z$ & $\left(\rho_{0} \rho_{1}\right)^{-1}$ & $\left(\rho_{1} \rho_{2}\right)^{\prime}$ & $\left(\rho_{2} \rho_{3}\right)^{-1}$ \\
\hline$\cdot 1$ & $736.10^{-7}$ & $2.10^{-8}$ & $<10^{-10}$ & $\cdot 9$ & .01698 & .00292 & $\cdot 00005$ \\
\hline$\cdot 4$ & .00248 & .00005 & $<10^{-7}$ & $1 \cdot 0$ & .02153 & $\cdot 00447$ & $\cdot 00011$ \\
\hline$\cdot 5$ & .004281 & .000156 & $2.10^{-7}$ & $1 \cdot 1$ & $\cdot 02667$ & $\cdot 00700$ & $\cdot 00024$ \\
\hline$\cdot 6$ & $\cdot 006629$ & $\cdot 000405$ & $10^{-6}$ & $1 \cdot 2$ & $\cdot 03223$ & $\cdot 00999$ & $\cdot 00051$ \\
\hline$\cdot 8$ & .01298 & $\cdot 00169$ & .00002 & $1 \cdot 5$ & $\cdot 05223$ & $\cdot 02311$ & $\cdot 00258$ \\
\hline
\end{tabular}
for $n=0,1,2,3, \ldots$.

TABLE 5.

The value of $\left(\rho_{\mathrm{g}} \rho_{\mathrm{t}}\right)^{-1}$ for $z=1.5$ is only $5.10^{-5}$, and it is therefore unnecessary in the above table.

TABLE 6.

\begin{tabular}{|c|c|c|c|c|c|c|}
\hline$z$ & 2 & $2 \cdot 5$ & 3 & 4 & 5 & 6 \\
\hline$\left(\rho_{0} \rho_{1}\right)^{-1}$ & $\cdot 09605$ & $\cdot 15274$ & $\cdot 22219$ & $\cdot 39938$ & $\cdot 6272$ & $\cdot 9058$ \\
\hline$\left(\rho_{1} \rho_{2}\right)^{-1}$ & $\cdot 05900$ & $\cdot 11107$ & $\cdot 17890$ & $\cdot 35207$ & $\cdot 5787$ & $\cdot 8564$ \\
\hline$\left(\rho_{2} \rho_{3}\right)^{-1}$ & 01575 & 04821 & $\cdot 10225$ & 26255 & $\cdot 4839$ & $\cdot 7591$ \\
\hline$\left(\rho_{3} \rho_{4}-1\right.$ & $\cdot 00104$ & $\cdot 00806$ & 03104 & $\cdot 1479$ & $\cdot 3498$ & $\cdot 6172$ \\
\hline$\left(\rho_{4} \rho_{5}\right)^{-1}$ & 00001 & 0003 & $\cdot 00329$ & $\cdot 0496$ & $\cdot 1971$ & $\cdot 4391$ \\
\hline$\left(\rho_{5} \rho_{6}\right)^{-1}$ & $<10^{-7}$ & $<10^{-5}$ & $<10^{-3}$ & $\cdot 0070$ & $\cdot 0708$ & $\cdot 2490$ \\
\hline$\left(\rho_{6} \rho_{7}\right)^{-1}$ & $<10^{-9}$ & $<10^{-7}$ & $<10^{-4}$ & $\cdot 00033$ & $\cdot 0122$ & $\cdot 0945$ \\
\hline
\end{tabular}

The extra values used are

(1) for $z=5$,

$$
\left(\rho_{7} \rho_{8}\right)^{-1}=\cdot 0008
$$

(2) for $z=6, \quad\left(\rho_{7} \rho_{8}\right)^{-1}=0187, \quad\left(\rho_{8} \rho_{9}\right)^{-1}=0016, \quad\left(\rho_{9} \rho_{10}\right)^{-1}=00006$.

If $S$ denotes the series

$$
S=\sum_{n=0}^{n=\infty}\left(\rho_{n} \rho_{n+1}\right)^{-1},
$$


the values of $S$ and of the pressure ratio $p / \frac{\pi}{*} a E$ are given in the final table, with the corresponding ratios of diameter to wave length. The pressure ratio is $6 \pi^{2} S / z^{3}$.

TABLE $\tau$.

\begin{tabular}{|c|c|c|c|c|c|c|c|}
\hline$z$ & $S$ & $\begin{array}{c}\text { Diameter } \\
\text { wave length }\end{array}$ & $p / \frac{8}{3} a E$ & $z$ & $S$ & $\begin{array}{c}\text { Diameter } \\
\text { wave length }\end{array}$ & $p / \frac{8}{3} a E$ \\
\hline$\cdot 1$ & $736.10^{-7}$ & $\cdot 032$ & $4 \cdot 358$ & $1 \cdot 2$ & $\cdot 04273$ & $\cdot 382$ & $1 \cdot 464$ \\
\hline$\cdot 4$ & $253.10^{-6}$ & $\cdot 127$ & $2 \cdot 342$ & $1 \cdot 5$ & $\cdot 07797$ & $\cdot 477$ & $1 \cdot 368$ \\
\hline$\cdot 5$ & $444.10^{-5}$ & $\cdot 159$ & $2 \cdot 102$ & 2 & $\cdot 1718$ & $\cdot 637$ & $1 \cdot 272$ \\
\hline$\cdot 6$ & $703 \cdot 5.10^{-5}$ & $\cdot 190$ & $1 \cdot 929$ & $2 \cdot 5$ & $\cdot 3204$ & $\cdot 796$ & $1 \cdot 214$ \\
\hline$\cdot 8$ & $\cdot 01469$ & $\cdot 255$ & $1 \cdot 699$ & 3 & $\cdot 5377$ & $\cdot 955$ & $1 \cdot 179$ \\
\hline$\cdot 9$ & $\cdot 01995$ & $\cdot 286$ & $1 \cdot 621$ & 4 & $1 \cdot 219$ & $1 \cdot 27$ & $1 \cdot 128$ \\
\hline $1 \cdot 0$ & $\cdot 02611$ & $\cdot 318$ & 1.546 & 5 & $2 \cdot 3205$ & $1 \cdot 59$ & $1 \cdot 099$ \\
\hline $1 \cdot 1$ & $\cdot 03392$ & $\cdot 350$ & $1 \cdot 509$ & 6 & 3.941 & $1 \cdot 91$ & 1.081 \\
\hline
\end{tabular}

The character of this table is somewhat unexpected. The ratio of the pressure to its limiting value for a large obstacle approaches the value unity in a regular manner as the size of the obstacle increases. This is especially evident if the values are plotted on a curve. The difference in behaviour of the cylinder and sphere is remarkable; for, in the present case, the pressure may be made greater than a bodily action, such as gravitation, in any arbitrarily assigned ratio by taking the radius of the cylinder small enough. This is, of course, only true in practice if a cylinder composed of so small a number of molecules could continue to approximate to a perfect reflector; but it is evident that the ratio can be made very great. The importance of this result to astrophysics is contained in the fact that the cylinder is, perhaps, a better type of obstacle than the sphere for the representation of the state of things which may obtain, for example, in the tail of a comet or in the solar corona.

In order to illustrate further the manner in which the pressure tends towards its limiting value, the case $z=8$ has been worked out in detail. The calculation by the previous method being very laborious in this case, another method was adopted. The functions $R_{0}, R_{1}$, and $R_{2}$ were calculated from the asymptotic series*

$$
R_{n}(z)=1+\frac{n^{2}-\frac{1}{4}}{z^{2}} \frac{1}{2}+\frac{\left(n^{2}-\frac{1}{4}\right)\left(n^{2}-\frac{9}{4}\right)}{z^{4}} \frac{1.3}{2.4}+\ldots
$$


and the corresponding values of $R_{3}, R_{4}, \ldots$, by the recurrence formula (33). The pressure is then given by

$$
p=\frac{4 a E}{z} \sum_{1}^{*}\left(R_{n} R_{n+1}\right)^{-1} .
$$

The results of the calculation are as follows:-

$$
\begin{aligned}
& R_{0}=998062, \quad R_{1}=1.005831, \quad R_{2}=1.021631, \\
& R_{3}=1.07016, \quad R_{4}=1.12982, \quad R_{5}=1.28546, \\
& R_{6}=1 \cdot 4568, \quad R_{7}=1 \cdot 7607, \quad R_{8}=2 \cdot 4709, \\
& R_{9}=4.3223, \quad R_{10}=17 \cdot 3521, \quad\left(R_{0} R_{1}\right)^{-1}=99613, \\
& \left(R_{1} R_{2}\right)^{-1}=\cdot 97315, \quad\left(R_{2} R_{3}\right)^{-1}=\cdot 91466, \quad\left(R_{3} R_{4}\right)^{-1}=\cdot 82708, \\
& \left(R_{4} R_{5}\right)^{-1}=\cdot 68854, \quad\left(R_{5} R_{6}\right)^{-1}=\cdot 53398, \quad\left(R_{6} R_{7}\right)^{-1}=\cdot 38987, \\
& \left(R_{7} R_{8}\right)^{-1}=\cdot 22985, \quad\left(R_{8} R_{9}\right)^{-1}=\cdot 09363, \quad\left(R_{0} R_{10}\right)^{-1}=\cdot 01333 \text {, }
\end{aligned}
$$

and the next function is of order $10^{-3}$.

The resulting value of the pressure is given by

$$
p / \frac{\kappa}{3} a E=1 \cdot 061,
$$

and corresponds to an obstacle whose diameter is 2.54 wave lengths. The approach towards the limiting value is still regular.

\section{PART II.}

When the incident magnetic force is parallel to the axis of the cylinder the analysis proceeds on very similar lines, although the final tabulation is somewhat more troublesome. In the present section of the paper this case is worked out in detail. It is then possible to construct the general case of incidence, of waves travelling perpendicular to the axis, without difficulty and to submit it to examination.

The analysis is given briefly, being analogous throughout to that of the first section. It is now the function $\phi$ which exists instead of $\psi^{\prime}$, and the radial, transversal, and axial components of the vectors are

$$
\left.\begin{array}{l}
a=0, \quad b=0, \quad c=-i \hbar \phi / V \\
P=-\partial \phi / r \partial \theta, \quad Q=\partial \phi / \partial r, \quad R=0
\end{array}\right\} .
$$

The magnetic force in the incident waves is again $e^{i k x}$, but in a new direction. The time factor is $e^{i k v t}$, and the corresponding electric force is $-V e^{i k x}$ perpendicular to the axis and to the incident ray. Its radial 
component is $-V \sin \theta e^{i l \cdot r \cos \theta}$, which, as in (1), may be expressed in the form

$$
P_{1}=-\frac{2 V}{k r} \Sigma_{1}^{\infty} i^{n-1} n \sin n \theta J_{n}(k r)
$$

with the magnetic force

$$
c=J_{0}(k r)+2 \Sigma_{1}^{\infty} i^{n} J_{n}(k r) \cos n \theta
$$

so that the function $\phi_{1}$ for the incident waves is given by

$$
\phi_{1}=\frac{i V}{k} J_{0}(k r)-\frac{2 V}{k} \Sigma_{1}^{\infty} i^{n-1} \cos n \theta J_{n}(k r) .
$$

With this we associate a formula involving the function $K_{n}(k r)$ instead of $J_{n}(k r)$, so that the functions together satisfy the surface condition. The magnetic force is necessarily tangential by the vanishing of $\psi$. The electric force will be normal if $\partial \phi / \partial r=0$. This is sufficient to secure the satisfaction of the surface conditions, and, determining the coefficients of the portion of $\phi$ representing the effect of the obstacle, the final form of $\phi$ for the external space is

$$
\begin{aligned}
\phi= & \frac{i V}{k}\left\{J_{0}(k r)-J_{0}^{\prime}(k a) K_{0}(k v) / K_{0}^{\prime}(k a)\right\} \\
& -\frac{2 V}{k} \Sigma_{1}^{\infty} i^{n-1} \cos n \theta\left\{J_{n}(k r)-J_{n}^{\prime}(k a) K_{n}(k v) / K_{n}^{\prime}(k v)\right\},
\end{aligned}
$$

or, in the notation of Part I,

$$
\begin{gathered}
\phi=\frac{i V}{k}\left\{J_{0}(k v) Y_{0}^{\prime}(k a)-Y_{0}(k r) J_{0}^{\prime}(k a)\right\} /\left\{Y_{0}(k a)+i \pi J_{0}(k a)\right\} \\
-\frac{2 V}{k} \Sigma_{1}^{\infty} i^{n-1} \cos n \theta\left\{J_{n}(k v) Y_{n}^{\prime}(k a)-Y_{n}(k r) J_{n}^{\prime}(k a)\right\} \\
\text { If } \\
\qquad Y_{n}^{\prime}(k a)+i \pi J_{n}^{\prime}(k a)=M_{n} e^{i \eta_{n}},
\end{gathered}
$$

the radial electric force at the surface is

or

$$
\begin{gathered}
P=-(\partial \phi / r \partial \theta)_{r=a} \\
\rho=-\frac{4 V}{k^{2} a^{2}} \Sigma_{1}^{\infty} \frac{n i^{n-1} \sin \theta}{M_{n}} e^{i k V t-i \eta_{n}}
\end{gathered}
$$

and the magnetic force is $c=-i k \phi / V$, or

$$
c=\frac{2}{k a I_{0}} e^{i k V t-i \eta_{0}}+\frac{4}{k a} \sum_{1}^{\infty} \frac{i^{i n} \cos n \theta}{M_{n}} e^{i l \cdot V t-i \eta_{n}}
$$

where the usual identical relation between the two types of Bessel function has been employed and the time factor has been inserted. Now the pressure $p$ acting on a unit length of the cylinder, derived as before 
from the resultant of the Maxwellian tractions on its outer surface, is given by

$$
p=E \iint\left(c^{2}-P^{2} / V^{2}\right) \cos \theta d S,
$$

the integral being taken over the curved surface, $E$ being the density of incident energy, and average values of the squares being taken. This is equivalent to a line integral round the circular section, and we obtain

$$
\frac{p}{2 a E}=\int_{0}^{\pi}\left(c^{2}-P^{2} / V^{2}\right) \cos \theta d \theta .
$$

The average values of the squares are

$$
\begin{aligned}
\overline{c^{2}}= & \frac{2}{k^{2} a^{2} M_{0}^{2}}+\frac{8}{k^{2} a^{2}} \Sigma_{1}^{\infty} \frac{\cos ^{2} n \theta}{M_{n}^{2}}+\frac{8}{k^{2} a^{2} M_{0}} \Sigma_{1}^{\infty} \frac{\cos n \theta}{M_{n}} \cos \left(\eta_{0}-\eta_{n}+n \frac{\pi}{2}\right) \\
& +\frac{16}{k^{2} a^{2}} \Sigma_{1}^{\infty} \Sigma_{1}^{\infty} \frac{\cos r \theta \cos s \theta}{M_{r} M_{s}} \cos \left\{\eta_{r}-\eta_{s}+(s-r) \frac{\pi}{2}\right\}, \\
\overline{P^{2} / V^{2}}= & \frac{8}{k^{4} a^{4}} \Sigma_{1}^{\infty} \frac{n^{2} \sin ^{2} n \theta}{M_{n}^{2}} \\
& +\frac{16}{k^{4} a^{4}} \Sigma_{1}^{\infty} \Sigma_{1}^{\infty} \frac{r \cdot s \sin r \theta \sin s \theta}{M_{r} M_{s}} \cos \left\{\eta_{r}-\eta_{s}+(s-r) \frac{\pi}{2}\right\},
\end{aligned}
$$

with equal values of $r$ and $s$ excluded from the double summations. With the relations

$$
\begin{aligned}
& \int_{0}^{\pi}\left(\cos ^{2} n \theta, \sin ^{2} n \theta\right) \cos \theta d \theta=0 \quad(n=0,1,2, \ldots), \\
& \int_{0}^{\pi} \cos n \theta \cos \theta d \theta=0 \quad(n \neq 1)=\frac{\pi}{2} \quad(n=1), \\
& \int_{0}^{\pi} \sin r \theta \sin s \theta \cos \theta d \theta=0 \quad(s \sim r \neq 1) \\
&=\frac{\pi}{4} \quad(s \sim r=1)
\end{aligned}
$$

we find $\int_{0}^{\pi}\left(\overline{P^{2} / V^{2}}\right) \cos \theta d \theta=\frac{4 \pi}{k^{4} a^{4}} \Sigma_{1}^{\infty} \frac{n(n+1)}{M_{n} \overline{M_{n+1}}} \sin \left(\eta_{n+1}-\eta_{n}\right)$,

$$
\int_{0}^{\pi} \overline{c^{2}} \cos \theta d \theta=\frac{4 \pi}{k^{2} a^{2}} \Sigma_{0}^{\infty} \frac{1}{M_{n} M_{n+1}} \sin \left(\eta_{n+1}-\eta_{n}\right),
$$

the summations starting at different points; but the first can also be 
regarded as starting at $n=0$. Thus, by (53), with $k a=z$ as usual,

$$
\frac{p}{2 a E}=\frac{4 \pi}{z^{2}} \sum_{n}^{\infty}\left(1-\frac{n . n+1}{z^{2}}\right) \frac{1}{M I_{n} M I_{n+1}} \sin \left(\eta_{n+1}-\eta_{n}\right) .
$$

But, by (49), $\quad \sin \left(\eta_{n+1}-\eta_{n}\right)=\pi\left(Y_{n}^{\prime} J_{n+1}^{\prime}-J_{n}^{\prime} Y_{n+1}^{\prime}\right) / M_{n} I I_{n+1}$,

and it is not difficult to establish the identity

$$
Y_{n}^{\prime}(z) J_{n+1}^{\prime}(z)-J_{n}^{\prime}(z) Y_{n+1}^{\prime}(z)=\frac{2}{z}\left(1-\frac{n . n+1}{z^{2}}\right),
$$

although it has apparently not been explicitly noticed. Accordingly,

$$
\sin \left(\eta_{n+1}-\eta_{n}\right)=\frac{2 \pi}{z M_{n} M_{n+1}}\left(1-\frac{n . n+1}{z^{2}}\right),
$$

and the pressure is finally given by

$$
\frac{p}{2 a E}=\frac{8 \pi^{2}}{z^{3^{-}}} \Sigma_{0}^{\infty}\left(1-\frac{n . n+1}{z^{2}}\right)^{2} /\left\{Y_{n}^{\prime 2}+\pi^{2} J_{n}^{\prime 2}\right\}\left\{Y_{n+1}^{\prime 2}+\pi^{2} J_{n+1}^{\prime 2}\right\},
$$

and this is the formula which must be tabulated.

\section{Case of a Large Obstacle.}

We must first show that this formula approximates to the correct result for a large obstacle when $z$ becomes large. This result is, of course, again $p=\frac{8}{3} a E$. The method employed in Part $I$ is again of service. We introduce the functions $R_{n}$ and $\phi_{n}$ of (29) and of the earlier paper. Then

$$
\begin{aligned}
M_{n}^{2} & =Y_{n}^{\prime 2}+\pi^{2} J_{n}^{\prime 2}=\frac{1}{4}\left\{\left(Y_{n-1}-Y_{n+1}\right)^{2}+\pi^{2}\left(J_{n-1}-J_{n+1}\right)^{2}\right\} \\
& =\frac{1}{4}\left\{\frac{2 \pi}{z} R_{n-1}+\frac{2 \pi}{z} R_{n+1}-\frac{4 \pi}{z}\left(R_{n-1} R_{n+1}\right)^{\frac{1}{t}} \cos \left(\phi_{n-1}-\phi_{n+1}\right)\right\} .
\end{aligned}
$$

But for a large value of $z$ successive functions of type $R$ will differ from one another by practically infinitesimal amounts, and thus, when they occur in the same term of an infinite series like (61), they may be equated. Accordingly, it is lawful to write

$$
M_{n}^{2}=\frac{\pi}{z} R_{n}\left[1-\cos \left(\phi_{n-1}-\phi_{n+1}\right)\right]
$$


But, under the same conditions,

$$
\phi_{n-1}-\phi_{n+1}=\left(\phi_{n-1}-\phi_{n}\right)+\left(\phi_{n}-\phi_{n+1}\right)=2\left(\phi_{n-1}-\phi_{n}\right),
$$

so that, by the recurrence formula (33),

$$
1-\cos \left(\phi_{n-1}-\phi_{n+1}\right)=2 \sin ^{2}\left(\phi_{n-1}-\phi_{n}\right)=2 / R_{n} R_{n-1}=2 / R_{n}^{2} \text {. }
$$

Finally, then, we may write in any single term of the series (61),

$$
M_{n}^{2} M_{n+1}^{2}=4 \pi^{2} / z^{2} R_{n}^{2}
$$

and therefore

$$
\frac{p}{2 a E}=\frac{2}{z} \Sigma_{0}^{\infty} R_{n}^{2}\left(1-\frac{n \cdot n+1}{z^{2}}\right)^{2}
$$

for a large obstacle. This process is, of course, only valid in the region of variation of $n$ such that $R_{n}$ does not become large. But there is no necessity to repeat the argument which shewed, in Part $I$, that the important part of the sum of series such as (61), when $z$ is large, is derived from the terms in which $n$ ranges from unity to a value differing from $z$ by a quantity of lower order. For this range $R_{n}$ is not large. We may therefore write, in order to obtain the first term of the pressure expressed as a series in descending order of $z$,

$$
\frac{p}{2 a E}=\frac{2}{z} \Sigma_{0}^{z} R_{n}^{2}\left(1-\frac{n \cdot n+1}{z^{2}}\right)^{2}
$$

and under the saine conditions, by (31),

$$
R_{n}^{2}=1 /\left(1-\frac{n^{2}}{z^{2}}\right)
$$

Replacing this summation by the asymptotically equivalent integral with $n / z=x$ as variable in the ordinary way,

$$
\frac{p}{2 a E}=2 \int_{0}^{1}\left(1-x^{2}\right) d x=\frac{4}{3}
$$

or $p=\frac{8}{3} a E$. The formula is therefore verified for a large obstacle.

\section{The Small Obstacle.}

As in Part I, we proceed now to a determination of the pressure on a cylinder of small section due to its scattering action on the waves. Attention may now be confined to the first term of (61), in which we may write, 
retaining only the main terms,

$$
\left.\begin{array}{ll}
Y_{1}(z)=-\frac{2}{z}, & Y_{0}^{\prime}(z)=-Y_{1}=\frac{2}{z} \\
Y_{1}^{\prime}(z)=\frac{2}{z^{2}}, & J_{0}^{\prime}(z)=J_{0}^{\prime}(z)=0
\end{array}\right\} ;
$$

and therefore

$$
\frac{p}{2 a E}=\frac{8 \pi^{2}}{z^{3}} / \frac{4}{z^{2}} \frac{4}{z^{4}}=\pi^{2} z^{3} / 2,
$$

or if $\lambda$ be the wave length $p=8 \pi^{5} a^{4} E / \lambda^{3}$.

In this case both the pressure itself and the ratio $p / \frac{8}{3} a E$ tend to zero with the radius of the obstacle in contradistinction to the result in Part I.

\section{Numerical Tables.}

We proceed now to a tabulation of the formula (61), the numerical work being somewhat heavier than before, but much facilitated by the intermediate tables of Part I.

Tables 8 and 9 give the values of the derivates of the Bessel functions which are necessary later. These have been calculated from the tables of the functions themselves ( 1 and 2 ) by means of the relations, for any argument $z$,

$$
\left.\begin{array}{l}
2 J_{n}^{\prime}=J_{n-1}-J_{n+1} \\
2 Y_{n}^{\prime}=Y_{n-1}-Y_{n+1}
\end{array}\right\} .
$$

Much labour is saved later by retaining the factor 2 which appears in these relations. The last figure is approximate. $J_{0}^{\prime}$ and $Y_{0}^{\prime}$ are respectively $-J_{1},-Y_{1}$. The tables have been checked by the identity (60).

\section{TABLE 8.}

\begin{tabular}{|c|c|c|c|c|c|c|}
\hline$z$ & $2 J_{\mathrm{i}}^{\prime}$ & $2 J_{2}^{\prime}$ & $2 J_{3}^{\prime}$ & $2 Y_{1}^{\prime}$ & $2 Y_{2}^{\prime}$ & $2 Y_{3}^{\prime}$ \\
\hline$\cdot 5$ & $\cdot 908$ & .240 & .030 & $15 \cdot 70$ & $127 \cdot 5$ & $>10^{3}$ \\
\hline$\cdot 6$ & $\cdot 868$ & $\cdot 282$ & .043 & $11 \cdot 26$ & $73 \cdot 61$ & $751 \cdot 3$ \\
\hline$\cdot 8$ & $\cdot 7705$ & $\cdot 359$ & .075 & $7 \cdot 137$ & $30 \cdot 91$ & $240 \cdot 0$ \\
\hline$\cdot 9$ & $\cdot 713$ & $\cdot 3915$ & .093 & $6 \cdot 131$ & $21 \cdot 69$ & $150 \cdot 6$ \\
\hline 1.0 & $\cdot 650$ & .4205 & $\cdot 110$ & $5 \cdot 463$ & $15 \cdot 84$ & $99 \cdot 3$ \\
\hline
\end{tabular}


TABLE: 9.

\begin{tabular}{|c|c|c|c|c|c|c|c|}
\hline$z$ & $1 \cdot 2$ & 1.5 & 2 & $2 \cdot 5$ & 3 & 4 & 5 \\
\hline $2 J_{1}^{\prime}$ & $\cdot 5118$ & $\cdot 2797$ & $-\cdot 1289$ & $-\cdot 4945$ & $-\cdot 7461$ & $-\cdot 7612$ & $-\cdot 2242$ \\
\hline $2 J_{!}^{\prime}$ & $\cdot 4654$ & $\cdot 4970$ & $\cdot 4478$ & $\cdot 2805$ & $\cdot 0300$ & $-\cdot 4962$ & $-\cdot 6924$ \\
\hline $2 J_{:}^{\prime}$ & $\cdot 1543$ & $\cdot 2204$ & $\cdot 3188$ & $\cdot 3723$ & $\cdot 3541$ & $\cdot 0830$ & $-\cdot 3446$ \\
\hline $2 J_{4}^{\prime}$ & $\cdot 0323$ & $\cdot 0593$ & $\cdot 1219$ & $\cdot 1971$ & $\cdot 2661$ & $\cdot 2981$ & $\cdot 1037$ \\
\hline $2 . J_{j}^{\prime}$ & .005 & $\cdot 012$ & $\cdot 0328$ & .0696 & $\cdot 1206$ & $\cdot 2320$ & $\cdot 2602$ \\
\hline $2 J_{6}^{\prime}$ & $<10^{-3}$ & $<10^{-2}$ & $<10^{-2}$ & $10^{-口}$ & $\cdot 0405$ & $\cdot 1169$ & $\cdot 2077$ \\
\hline $2 Y_{1}^{\prime}$ & $4 \cdot 686$ & $4 \cdot 131$ & $3 \cdot 543$ & $2 \cdot 763$ & $1 \cdot 688$ & $-\cdot 7315$ & $-2 \cdot 124$ \\
\hline $2 Y_{2}^{\prime}$ & $9 \cdot 330$ & $5 \cdot 220$ & $3 \cdot 208$ & $2 \cdot 834$ & $2 \cdot 712$ & $1 \cdot 822$ & $\cdot 005$ \\
\hline $2 Y_{:}^{\prime}$ & $48 \cdot 45$ & $20 \cdot 20$ & $6 \cdot 75$ & $3 \cdot 302$ & $2 \cdot 376$ & $2 \cdot 214$ & $1 \cdot 759$ \\
\hline $2 Y_{4}^{\prime}$ & 327 & $110 \cdot 3$ & $27 \cdot 68$ & $9 \cdot 66$ & $4 \cdot 30$ & $1 \cdot 928$ & 1.886 \\
\hline $2 Y_{5}^{\circ}$ & $>10^{3}$ & 732 & $138 \cdot 7$ & $39 \cdot 12$ & $14 \cdot 20$ & $3 \cdot 179$ & $1 \cdot 643$ \\
\hline $2 r_{i j}^{\prime \prime}$ & $>10^{4}$ & $>10^{3}$ & $10^{3}$ & $2.10^{2}$ & $56 \cdot 3$ & $9 \cdot 145$ & $2 \cdot 542$ \\
\hline
\end{tabular}

The following additional values were employed :-

$$
\begin{aligned}
& \text { (1) } \quad z=4, \quad 2 J_{\uparrow}^{\prime}=\cdot 015, \quad 2 Y_{\mathrm{i}}^{\prime}=31 \cdot 33, \quad 2 Y_{\mathrm{s}}^{\prime}=120.9 \text {; } \\
& \text { (2) } \quad z=5, \quad 2 J_{\uparrow}^{\prime}=\cdot 113, \quad 2 J_{\Xi}^{\prime}=\cdot 048, \quad 2 J_{9}^{\prime}=\cdot 017 \text {, } \\
& 2 Y_{i}^{\prime}=6 \cdot\left(i 16, \quad 2 Y_{3}^{\prime}=20 \cdot 43, \quad 2 Y_{!}^{\prime}=78 \cdot 96, \quad 2 Y_{10}^{\prime}=302 \cdot 5\right. \text {. } \\
& \text { Let } \\
& \sigma_{u}=4\left\{\left(Y_{n}^{\prime}\right)^{2}+\pi^{2}\left(J_{n}^{\prime}\right)^{2}{ }_{i} .\right.
\end{aligned}
$$

'I'hen the values of $\sigma_{i}$ are exhibited in the next two tables:-

TABI, 10.

\begin{tabular}{|c|c|c|c|c|c|}
\hline$z$ & $\cdot 5$ & $\cdot 6$ & $\cdot 8$ & $\cdot 9$ & $1 \cdot 0$ \\
\hline$\sigma_{0}$ & $87 \cdot 80$ & $65 \cdot 96$ & $43 \cdot 144$ & $36 \cdot 604$ & $31 \cdot 732$ \\
\hline$\sigma_{1}$ & $254 \cdot 6$ & $134 \cdot 2$ & $56 \cdot 80$ & $42 \cdot 61$ & $34 \cdot 014$ \\
\hline$\sigma_{2}$ & 16256 & 5419 & $956 \cdot 7$ & $472 \cdot 0$ & $252 \cdot 6$ \\
\hline$\sigma_{3}$ & $>10^{6}$ & $564.10^{*}$ & 57600 & 22680 & 9861 \\
\hline
\end{tabular}


TABLE: 11.

\begin{tabular}{|c|c|c|c|c|c|c|c|}
\hline$z$ & $\sigma_{0}$ & $\sigma_{1}$ & $\sigma_{\underline{z}}$ & $\sigma_{3}$ & $\sigma_{4}$ & $\sigma_{5}$ & $\sigma_{G}$ \\
\hline $1 \cdot 1$ & 28.00 & $28 \cdot 425$ & $145 \cdot 2$ & 4657 & $25.10^{4}$ & $>10^{\mathrm{s}}$ & $>10^{12}$ \\
\hline $1 \cdot 2$ & 25.03 & 24.54 & $89 \cdot 19$ & 2348 & $10^{5}$ & $>10^{7}$ & $>10^{10}$ \\
\hline 1.5 & $19 \cdot 00$ & $17 \cdot 84$ & $29 \cdot 69$ & $408 \cdot 5$ & 12170 & $>10^{5}$ & $>10^{s}$ \\
\hline 2 & $13 \cdot 58$ & $12 \cdot 72$ & $12 \cdot 27$ & $46 \cdot 57$ & $766 \cdot 3$ & 19240 & $>10^{\circ}$ \\
\hline $2 \cdot 5$ & 10.596 & 10.048 & $8 \cdot 808$ & $12 \cdot 27$ & $93 \cdot 70$ & 1530 & $>4.10^{+}$ \\
\hline 3 & $8 \cdot 700$ & $8 \cdot 343$ & $7 \cdot 364$ & $6 \cdot 883$ & $19 \cdot 19$ & $201 \cdot 8$ & 3170 \\
\hline 4 & 6.424 & 6.254 & $5 \cdot 750$ & 4.970 & $4 \cdot 594$ & $10 \cdot 637$ & 83.77 \\
\hline 5 & $5 \cdot 100$ & 5.007 & $4 \cdot 732$ & $4 \cdot 266$ & 3.663 & $3 \cdot 368$ & 6.888 \\
\hline
\end{tabular}

The following are additional values which were required :-

$$
\begin{array}{rlrl}
\text { (1) } z & =4, \quad \sigma_{7}=981 \cdot 6, & \sigma_{8} & =15.10^{3} \\
\text { (2) } z & z=5, \quad \sigma_{7}=43 \cdot 90, & \sigma_{8} & =417 \cdot 3 \\
\sigma_{9} & =6234, & \sigma_{10} & =91500 .
\end{array}
$$

With:these values of the $\sigma$ 's the pressure is given from (61) by

$$
\frac{p}{2 a E}=\frac{12 n \pi^{2}}{z^{3}} \Sigma_{0}^{\infty}\left(1-\frac{n \cdot n+1}{z^{2}}\right)^{2} / \sigma_{n} \sigma_{n+1}
$$

We may denote $\left(\sigma_{n} \sigma_{n+1}\right)^{-1}$ ly $\tau_{n}$. 'l'hen the requisite values of $\tau_{n}$ are given in the next tables.

T.A][T]: 12.

\begin{tabular}{|c|c|c|c|c|c|}
\hline$z$ & 5 & $\cdot 6$ & $\cdot S$ & $\cdot 9$ & $1 \cdot 0$ \\
\hline$\tau_{0}$ & $4 \cdot 474.10^{-5}$ & $1 \cdot 130.10^{-4}$ & $4 \cdot 081.10^{-4}$ & $6 \cdot 480.10^{-4}$ & $9 \cdot 263.10^{-4}$ \\
\hline$\tau_{1}$ & $2 \cdot 416.10^{-7}$ & $1 \cdot 375.10^{-4}$ & $1 \cdot 840.10^{-3}$ & $4 \cdot 972.10^{-}$ & $1 \cdot 164.10^{-4}$ \\
\hline$\tau_{3}$ & $<10^{-11}$ & $3 \cdot 269.10^{-10}$ & $1 \cdot 815.10^{-8}$ & $9 \cdot 342.10^{-8}$ & $4 \cdot 016.10^{-7}$ \\
\hline
\end{tabular}


TABLE 13.

\begin{tabular}{|c|c|c|c|c|c|c|c|c|}
\hline$z$ & $1 \cdot 1$ & $1 \cdot 2$ & $1 \cdot 5$ & 2 & $2 \cdot 5$ & 3 & 4 & 5 \\
\hline$\tau_{0}$ & $1 \cdot 257.10^{-3}$ & $1 \cdot 628 \cdot 10^{-3}$ & $2 \cdot 950.10^{-3}$ & $5 \cdot 787.10^{-3}$ & $2 \cdot 396 \cdot 10^{-3}$ & $\cdot 01378$ & $\cdot 02489$ & .03916 \\
\hline$\tau_{1}$ & $2 \cdot 422.10^{-4}$ & $4 \cdot 569.10^{-4}$ & $1 \cdot 888.10^{-3}$ & $6 \cdot 407 \cdot 10^{-3}$ & $1 \cdot 130.10^{-2}$ & $1 \cdot 628.10^{-2}$ & $\cdot 02781$ & .04220 \\
\hline$\tau_{2}$ & $1.478 .10^{-6}$ & $4 \cdot 775.10^{-6}$ & $8 \cdot 246.10^{-5}$ & $1 \cdot 750.10^{-3}$ & $3 \cdot 253.10^{-3}$ & $1 \cdot 973.10^{-2}$ & $\cdot 03499$ & .04954 \\
\hline$\tau_{3}$ & $8 \cdot 6.10^{-10}$ & $4.10^{-3}$ & $2 \cdot 012.10^{-7}$ & $2 \cdot 802.10^{-5}$ & S.698.10 $10^{-4}$ & $7 \cdot 571.10^{-3}$ & $\cdot 04380$ & .06399 \\
\hline$\tau_{4}$ & $<10^{-13}$ & $<10^{-12}$ & $<10^{-10}$ & $7.10^{-s}$ & $6 \cdot 975.10^{-6}$ & $2.583 .10^{-4}$ & $\cdot 02046$ & $\cdot 08106$ \\
\hline$\tau_{5}$ & $<10^{-19}$ & $<10^{-18}$ & $<10^{-15}$ & $<10^{-10}$ & $<10^{-9}$ & $1 \cdot 563.10^{-6}$ & $1 \cdot 122.10^{-3}$ & .04311 \\
\hline
\end{tabular}

Additional values subsequently used are :-

(1) $z=4, \quad \tau_{6}=1 \cdot 216.10^{-j}, \quad \tau_{7}=6 \cdot 97.10^{-8}$;

(2) $z=5, \quad \tau_{6}=3.31 .10^{-3}, \quad \tau_{7}=5.46 .10^{-5}, \quad \tau_{8}=3.84 .10^{-i}$,

$$
\tau_{9}=1 \cdot 75 \cdot 10^{-9} \text {. }
$$

T'he final values of $p / \frac{8}{3} a E$, calculated from these data, by the formula

$$
p / \frac{8}{3} a E=\frac{96 \pi^{2}}{z^{3}} \Sigma_{1}^{\infty}\left(1-\frac{n \cdot n+1}{z^{2}}\right)^{2} \tau_{n},
$$

are shewn in the last table.

\begin{tabular}{|c|c|c|c|c|c|c|c|c|c|c|c|c|c|}
\hline$z$ & $\cdot 5$ & $\cdot 6$ & $\cdot 8$ & $\cdot 9$ & 1.0 & $1 \cdot 1$ & $1 \cdot 2$ & $1 \cdot 5$ & $2 \cdot 0$ & $2 \cdot 5$ & $3 \cdot 0$ & $4 \cdot 0$ & $5 \cdot 0$ \\
\hline $\begin{array}{l}\text { Diameter } \\
\text { wave length }\end{array}$ & $\cdot 159$ & $\cdot 190$ & $\cdot 255$ & $\cdot 286$ & $\cdot 318$ & $\cdot 350$ & $\cdot 382$ & $\cdot 477$ & $\cdot 637$ & $\cdot 796$ & $\cdot 955$ & $1 \cdot 27$ & $1 \cdot 59$ \\
\hline$p / \frac{8}{3} a E$ & $\cdot 429$ & $\cdot 621$ & .898 & $\cdot 987$ & $\cdot 998$ & $\cdot 985$ & $\cdot 957$ & $\cdot 900$ & $\cdot 940$ & $\cdot 934$ & .950 & $\cdot 946$ & .965 \\
\hline
\end{tabular}

TABLE 14.

The table shews that the pressure ratio exhibits a series of maxima and minima while tending towards the value unity on the whole. The principal maximum occurs near $z=1$, where the ratio is almost exactly unity. This series of maxima and minima is analogous to that which occurs in the case of the sphere which is not perfectly reflecting. It could have been predicted from the type of the formula (61), in which the variation of $z^{3} R_{n} R_{n+1}$ with $z$ is, for any value of $n$, of the nature of a continuous increase with $z$. The values become smaller rapidly as $n$ increases when $z$ is small, but if $z$ be larger they tend to the same order of magnitude. 
The pressure of radiation on a cylindricil obstacle. [Dec. 14,

Meanwhile the factor $\left(1-n . n+1 / z^{2}\right)^{2}$ vanishes whenever

$$
z^{2}=n . n+1,
$$

and obliterates the corresponding term, and a definite minimum of the pressure ratio follows. As $z$ increases, the obliterated term is otherwise a smaller fraction of the total, and the minima become less pronounced. The curve of the pressure ratio resembles a wave form of decaying amplitude tending asymptotically to a straight line. The table indicates this character clearly.

A combination of the two solutions yields the solution for the general two-dimensional problem. For, if the incident magnetic force $H$ makes any angle $\beta$ with the axis, it has components (1) $H \cos \beta$ along the axis, and (2) $H \sin \beta$ perpendicular to the axis and to the direction of propagation. These may be considered separately as in Parts I and II. If for a given wave length and diameter the pressure ratio $p / \frac{8}{3} a E$ becomes $A$ in the case of Part I and $B$ in that of Part II, the pressure in the general case must be

$$
p=\left(A \sin ^{2} \beta+B \cos ^{2} \beta\right) \frac{8}{3} a E .
$$

This appears at once from the nature of the analysis. Maxima and minima of this quantity, as a function of $\beta$, can only occur when $\beta$ is zero or $90^{\circ}$; that is to say, they occur in the two cases investigated in detail.

It follows that the cylinder can give extended support to the astrophysical theory based on radiation pressure, but only by virtue of the case discussed in Part I, and cases not too far removed from it. Here it gives valuable support in accordance with the brief discussion given in Part I. 